\title{
Internal and external characteristics of a superheated jet
}

\author{
A. Sinha, S. Balasubramanian \& S. Gopalakrishanan \\ Department of Mechanical Engineering, \\ Indian Institute of Technology, India
}

\begin{abstract}
Flashing phenomenon occurs by rapid release of sub cooled/saturated liquid into a medium with lower pressure. This creates an expansion associated with vapour formation. The thermodynamic instability causes breakup of the liquid jet. Understanding the physics behind the jet disintegration and flashing phenomenon is still an open problem, with applications in automotive and aerocombustor industry. The behaviour of a flashing jet is highly dependent on the input parameters, most importantly inlet temperature and pressure. In the present study, the external (outside nozzle) and the internal (inside nozzle) flow characteristics of the two-phase flow has been studied numerically and experimentally. The phase change from liquid to vapour takes place over a finite period of time. Homogenous Relaxation Model (HRM) is used to model such finite rate process. In order to validate the numerical results, controlled experiments were carried out. Results revealed that the mass flow rate obtained numerically matches with that of experiments. Optical diagnostics were used to study the flow characteristics. Flow characteristics in terms of spray angle, penetration depth will be obtained from the experiments for better understanding of the break-up mechanism involved.
\end{abstract}

Keywords: superheated, jet, Homogenous Relaxation Model, instability, aerodynamic, flashing, phase change, helical, axisymmetric, breakup.

\section{Introduction}

Superheated liquid when suddenly released under ambient conditions into the atmosphere, results into a formation of two-phase mixture. This phenomenon is mainly is due to the thermodynamic non-equilibrium of the liquid. The non- 
equilibrium is due to the higher temperature of the liquid above its saturation temperature at that particular pressure. Thermodynamic non-equilibrium state can be attained by two different routes: a) the fluid in liquid state and complete equilibrium is heated at constant pressure; and b) keeping the initial temperature constant, depressurize the liquid rapidly, this will result in the increase in the liquid internal temperature above the saturation temperature due to thermal inertia. In a cold axisymmetric jet, the breakup is mainly due to the shear between the liquid and the surrounding. This is type of breakup is referred as 'aerodynamic break up'. But in a superheated jet, along with aerodynamic phenomena, breakup can also occur due to formation of vapour which may burst out from the core of the jet to outside. This type of break up is referred to as flashing (Witlox [1]). Fig. 1 shows the characteristics of a superheated jet with the assumption that flashing phenomenon has started inside the nozzle. Different sections are levelled (A to D) to point out regions which influence the behaviour of flashing jet. Section A represents influence of all the inlet conditions like pressure, temperature along with the size, shape and roughness of the nozzle. Section B shows the path and manner the fluid is travelling inside the nozzle. Here, bubbles will start forming due to early nucleation. This is because inside the nozzle, the boiling phenomenon dominates. The velocity of the liquid along with bubble generation formed by boiling governs the nature of the flow. Section $\mathrm{C}$ shows the phenomenon outside the nozzle due to surface instability caused by aerodynamic and flashing mechanisms which influence the flow structure. Section D represents that in the far field the jet breakup with big droplets and ligaments breaking down into small droplets. This complex phenomenon is governed by evaporation and drag forces.

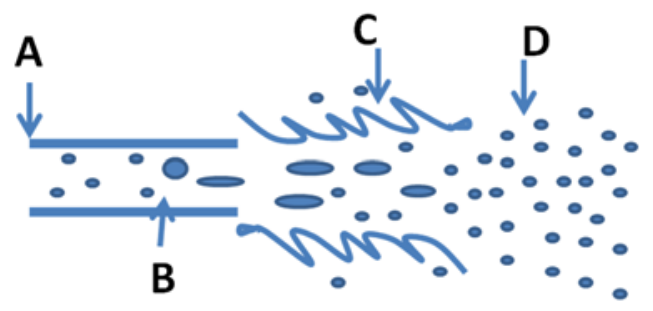

Figure 1: Factors influencing the characteristics of a superheated jet.

It is important to study the phase change process due to the increase in the popularity of direct injection system (Schmidt et al. [2]). The fluid in such system absorbs heat from the surrounding (relatively at higher temperature), i.e. inside the nozzle, before entering the combustion chamber or during compression in the pump, results in increase of its vapour pressure and temperature. If the temperature is more than the saturated temperature, the fluid will be in state of flash boiling. It is therefore important to understand the physics of the flow before it gets atomized into the combustion chamber. Also, to increase the performance of military aircrafts, the proposition to use the jet fuel as the heat sink, may act as a boon on the combustor efficiency and thermal 
management. It has been indicated by the system analysis that in order to meet the future heat load requirement, the jet fuel temperature needs to be increased beyond $370^{\circ} \mathrm{C}$ (Schulz [3], Spadaccini and Huang [4]). This high temperature of the fuel greatly increases the chances for the fuel, initially in liquid phase to be in both liquid and vapour phase - superheated state. This may result in vapour lock inside the nozzle. The characteristics and behaviour of these two-phase mixtures can also cause a potential hazard to the industrial environment (Gopalakrishnan [5], Allen [6]).

There are many factor on which the behaviour and formation of a superheated jet depends. Most importantly it depends on the inlet temperature and difference between internal and external pressure. Experiments were done using photography technique, Laser Doppler anemometry, in the past but failed to explain properly the phenomenon behind such complex flows (Polanco et al. [7]). There have been a few attempts to model such two-phase flows inside a nozzle numerically but they were mainly limited to one-dimensional flow (Barret et al. [8]). Though superheated jets have been studied adequately in the past but fundamental understanding of the physics behind such non-equilibrium twophase flow is still an open problem (Polanco et al. [7]). Moreover experimental data is not easy to obtain due to the complex nature of the flow and high pressure and temperature involved.

Any investigation in the physics of the superheated jet consists of two distinct sections - the internal nozzle flow and the external flow outside the nozzle. The external flow throws light on the development, structure and break of the jet. But the internal flow answers important questions about the amount of vapour formation inside the nozzle, the velocity at the exit of the nozzle and the effect of inlet pressure.

If time scale over which the phase change of the superheated fluid takes place inside the nozzle is longer compare to the flow though the nozzle, i.e. the residence time of the superheated fluid inside the nozzle is long compared to the relaxation time of the fluid, then the flow regime would be in a state of equilibrium. But, it may also happen that the time scale over which the phase change takes place is relatively small comparable to the flow through the time of the superheated fluid through the nozzle, then it will result in excessive vapour formation and may dramatically decrease the flow, which may result into a subsonic two-phase chocking inside the nozzle. Since the time scale of the flow through the nozzle is comparable to time over which the phase change occurs, it is necessary to model this phenomenon as a finite rate process. Homogenous Relaxation Model (HRM) has been employed to model the phase change of the fluid from liquid to vapour phase.

This works deals with the implementation of the HRM, to model the nonequilibrium finite rate phase change process. The HRM model is used to accurately predict the amount of mass flow rate outside the nozzle exit. As a first step, HRM is used without coupling any atomization model. Also, water is used as the working fluid to avoid complexity. Experiments were carried out with superheated water to validate the model and to understand the flow physics of the external jet. 


\section{Governing equations}

The mass conservation, momentum conservation and phase change model governs the flow physics. The symbol $\phi$ and $\tau$ in the following equations represents mass flux and the stress tensor respectively.

Continuity equation:

$$
\frac{\partial \rho}{\partial t}+\nabla \cdot \phi=0
$$

Momentum Conservation:

$$
\frac{\partial \rho U}{\partial t}+\nabla \cdot(\phi U)=-\nabla p+\nabla \cdot \overline{\bar{\tau}}
$$

The discretised momentum equation applied to each cell of the numerical domain is given in eqn. 3 , more details about this discretization can be found in (Ferziger and Peric [9]).

$$
a_{p} U_{p}=H(U)-(\nabla p)_{p}
$$

where subscript $\mathrm{p}$ refers to the cell in the computational domain, $U$ is the velocity and $\mathrm{H}$ represents the operator of summing the diffusion and convection terms and $a_{p}$ is the diagonal of the coefficient matrix of the velocity equation.

The energy equation used by Gopalakrishnan [5] is used here. The energy equation is added neglecting the kinetic energy of the fluid, conduction and viscous energy dissipation.

$$
\frac{\partial(\rho h)}{\partial t}+\nabla \cdot(\phi \mathrm{h})=\frac{\partial \mathrm{p}}{\partial \mathrm{t}}+\mathrm{U} \cdot \nabla \mathrm{p}
$$

The above equations are not in closed form. The two-phase mixture is not in thermal equilibrium. Therefore, HRM is used.

\subsection{Homogenous Relaxation Model}

HRM is modelled as a first order system. The model represents the complex process through which two phases exchange heat and mass. The model determines the total derivative of quality (mass fraction of vapour) as given in eqn. 5 .

HRM in mathematical form is:

$$
\frac{d x}{d t}=\frac{x^{e q}-x}{\theta}
$$

Eqn. 5 shows the exponential relationship of the equilibrium quality $\left(x^{e q}\right)$ to the quality $(\mathrm{x})$, over a time scale $(\theta)$. The time scale is based on an empirically fit to experimental data based on a flashing flow in water in a straight, smooth, long pipe. This is based on the work by Downar-Zapolski et al. [10]. The correlation is based on best-fit values of experimental data, given in eqn. 6 . The parameters include $\theta_{0}$ and two exponential components.

$$
\theta=\theta_{0} \alpha^{a} \beta^{b}
$$


where the values of $\alpha=-0.54$ and $\beta=-1.76$.

The two variables $\alpha$ and $\beta$ represents the volume fraction of vapour and the dimensionless pressure difference between the local pressure $(\mathrm{P})$ and saturated pressure $\left(\mathrm{P}_{\mathrm{s}}\right)$ as shown in eqn. 7 respectively

$$
\beta=\left|\frac{P-P s}{P_{S}}\right|
$$

The value $\theta_{0}$ used in the present work is different to that given by DownarZapolski. This is because the value of $\theta_{0}\left(6.51 \times 10^{-4} \mathrm{~s}\right)$ given by Downar is for flow in a pipe. It is observed numerical trials that for external flow this value doesn't suit well. The empirical value considered based on numerical trials is $6.51 \times 10^{-7} \mathrm{~s}$.

The HRM is coupled with mass, momentum and energy conservation though the dependence of fluid properties. PISO algorithm is implemented to update the pressure field from the velocity field predicted using lagged pressure as shown in eqn. 8 .

$$
p \nabla \cdot\left(\frac{H}{a_{p}}\right)-\rho \nabla a_{p} \nabla p^{k+1}+\sigma\left(p^{k}\right)+\frac{\partial \sigma}{\partial p}\left(p^{k+1}-p^{k}\right)=0
$$

This will act as a corrector to velocity field as shown in eqn. 9.

$$
\frac{\partial \rho U^{0}}{\partial t}+\nabla\left(\phi U^{0}\right)=-\nabla p^{n}+\nabla \mu \nabla U^{0}
$$

\section{Experimental set-up}

In order to simplify the geometrical system, the experiments were conducted on cylindrical jet oozing out horizontally from a round nozzle. A schematic diagram of the experimental set-up is shown in fig. 2. It consists of a high pressure cylindrical stainless steel tank of diameter $200 \mathrm{~mm}$ and a height of $150 \mathrm{~mm}$. The tank is equipped with a $2 \mathrm{~kW}$ heater, which is mounted coaxially with the inlet of the nozzle. The nozzle used in this study is of $10 \mathrm{~mm}$ length and $5 \mathrm{~mm}$ diameter. This length is enough to have a fully developed profile at the nozzle exit. The nozzle opens into large glass tank $(600 \mathrm{~mm}$ x $600 \mathrm{~mm})$ filled with homogenous fluid; the jet external characteristics were measured as the jet exits into the large glass tank. An outflow arrangement is made to prevent flow reversal and to maintain a constant head in the tank. The effect of surface roughness on the nozzle was not considered as it is made of glass with smooth surface texture. The fluid used in the present study is water. Though the maximum withstanding pressure of the tank is 3 bar, but this was not employed in the present study due to practical difficulties. Thermocouples of $\mathrm{K}$ type were used to measure the instantaneous temperature in the system. Two thermocouples were placed along the circumference of the tank and one thermocouple was placed at exit of the nozzle to record the exit temperature, which was later used to measure the mass flow rate for validation with numerical results. In order to measure pressure inside the system, a bourdon type stainless steel pressure gauge was installed on the top of the tank. A flow control valve is used for controlling smooth flow of the fluid through the nozzle. For safety, a pressure relief was mounted on the top of the tank. 


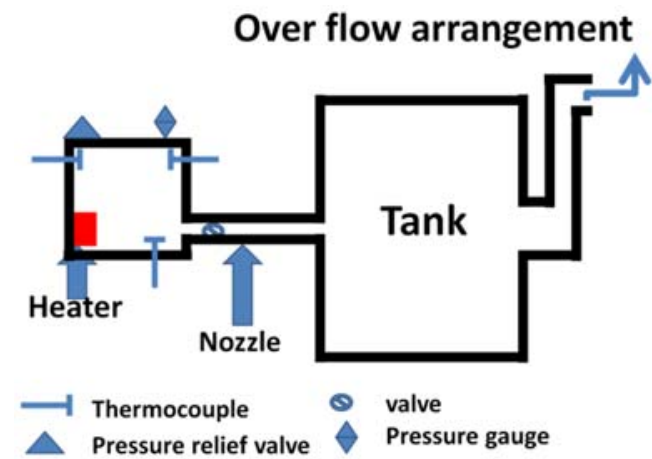

Figure 2: Schematic diagram of the experimental set-up.

\section{Code validation and results}

All the numerical simulations and experimental test cases were done using water. Simulations are carried out until the flow reached a stable state. This is because the experimental results were always in a quasi-steady state. For numerical easiness, the present flow was simulated as a 2-D flow. A plenum was added both at the inlet and the outlet to prevent imposing boundary conditions as shown in fig. 3. This will avoid boundary condition where sharp gradient is present. Also the presence of plenum will provide some region between the region of interest and imposed boundary condition. A structured mesh was used throughout the whole domain. The number of cells used is 1,000,000 after grid convergence. The height of the domain numerical is $55 \mathrm{~mm}$. The other dimensions of the computational domain are shown in fig. 3. All the dimensions are in $\mathrm{mm}$.

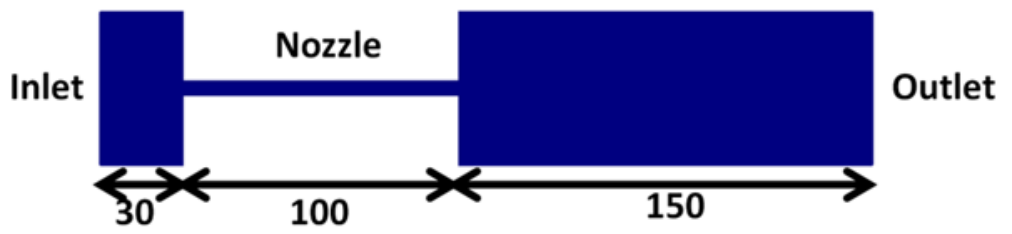

Figure 3: Two dimensional numerical domain.

The numerical domain was meshed using hexahedral cells. Fixed pressure boundary conditions were imposed at the inlet and the outlet. The walls were maintained at zero velocity and zero pressure gradients. The diameter of the nozzle is $5 \mathrm{~mm}$ and the length of the nozzle is $180 \mathrm{~mm}$. This length is necessary to have a full developed jet at the nozzle exit. The outlet pressure is maintained at 1 bar for all the numerical cases. After the nozzle, the jet comes into an open 
atmosphere filled with water. There are no walls after the nozzle outlet. Walls are present only before the nozzle and at the nozzle boundary.

\subsection{Validation}

Experiments were conducted using an axisymmetric nozzle. The downstream pressure was maintained at 1 bar. For validation purpose, the temperature and pressure is varied at the inlet and compared with that in the numerical simulations. The pressure considered only for validation purpose are 2.5 bar, 2.0 bar and 1.8 bar at $403 \mathrm{~K}, 398 \mathrm{~K}$ and $396 \mathrm{~K}$ respectively. As shown in table 1 , the mass flow rate obtained experimentally matches with the numerical result well within the error limit.

Table 1: Mass flow rate comparison between numerical and experimental results.

\begin{tabular}{ccccc}
$\begin{array}{c}\text { Inlet } \\
\text { temperature } \\
\text { (K) }\end{array}$ & $\begin{array}{c}\text { Pressure } \\
\text { (bar) }\end{array}$ & $\begin{array}{c}\text { Mass flow rate } \\
\text { (numerical, Kg/s) }\end{array}$ & $\begin{array}{c}\text { Mass flow rate } \\
\text { (experiment, Kg/s) }\end{array}$ & $\begin{array}{c}\text { \% difference } \\
\text { (error) }\end{array}$ \\
\hline 403 & 2.5 & 0.0166 & 0.0155 & 8.2 \\
\hline 398 & 2.0 & 0.0235 & 0.0222 & 6.5 \\
\hline 396 & 1.8 & 0.0180 & 0.0207 & 13.4 \\
\hline
\end{tabular}

\subsection{Numerical results}

The pressures considered for numerical analysis are 2 bar and 5 bar at fixed inlet temperature of $393 \mathrm{~K}$. This is because the pressures and temperatures used in validation are not large enough to cause to huge difference in the mass fraction, density or velocity for comparison. The temperature of the fluid at the inlet was kept below the saturation temperature at that particular pressure, in order to maintain a sub-cooled state of the liquid at the inlet of the numerical domain. The liquid accelerates as it flows through the nozzle due to drop in pressure. Once the local pressure of the liquid falls below the saturation pressure, the liquid starts to flash boil. The acceleration of the liquid increases with the increase in pressure ratio i.e. inlet to outlet pressure, as shown in velocity contour fig. 4 and fig. 5 at time $=0.02$ second. The unit in the contour is $\mathrm{m} / \mathrm{s}$.

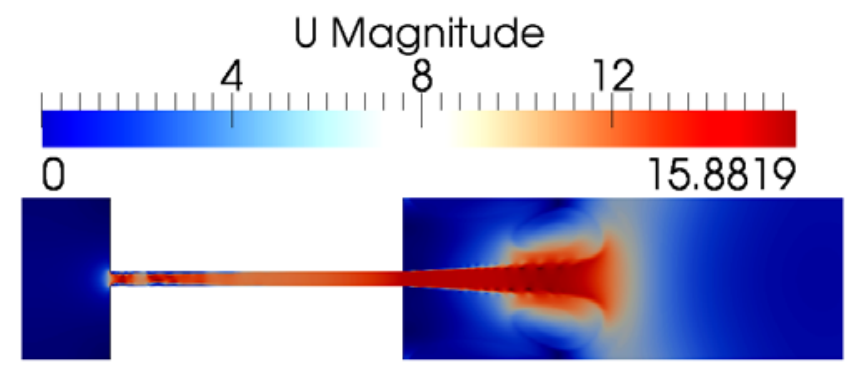

Figure 4: Velocity contour for $\mathrm{P}=2$ bar. 
Once the jet comes out of the nozzle, due to the sudden interaction with the surrounding fluid, a little disturbance is observed as shown in fig. 4 . The shear stress between the jet and the ambient fluid causes instability in the jet, which grows downstream of the nozzle with the development of axisymmetric structure. The two vortical structures which are observed downstream of the nozzle in fig. 4 are due to shear formation of the jet and the surrounding fluid. The jet expands with the increase in axial distance due to the entrainment of the surrounding fluid.

In fig. 5 acceleration of the jet is more due to the higher inlet to outlet pressure ratio. The most significant observation in fig. 5 is the formation of a helical structure, which grows as the jet moves downstream. This is due to higher formation of vapour, which causes increase in the velocity magnitude of the jet.

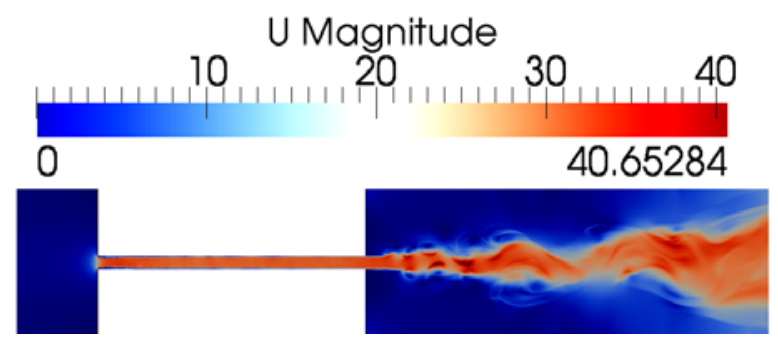

Figure 5: Velocity contour for $\mathrm{P}=5$ bar.

The phase changes process i.e. vapourization, start at the inlet corner of the nozzle at the formation of vena contracta as shown in fig. 6 and fig. 7. This is due to the separation of the flow at the sharp inlet of the nozzle, which causes a decrease in the local pressure at that particular location below the saturation pressure. This shows that similar to cavitations, flashing phenomenon can also be geometrically induced. The axisymmetric structure i.e. contraction and expansion of the jet is clearly observed in fig. 6 .

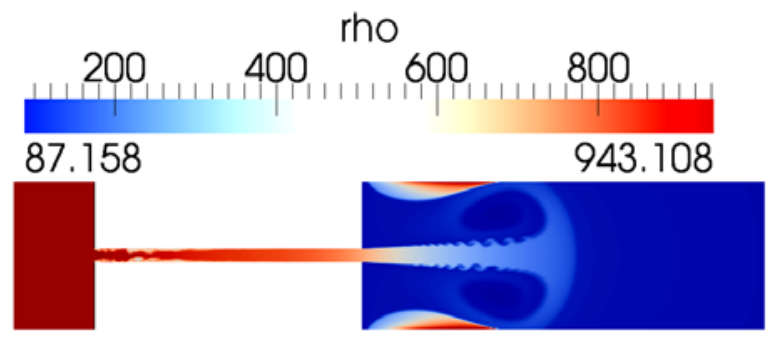

Figure 6: Density contour for $\mathrm{P}=2$ bar.

The increase in pressure ratio from 2 to 5 creates more vapour formation, which can be observed with the reduction in the density of the fluid as shown in fig. 7. 
It is also observed in fig. 7 that mode of instability changes from axisymmetric to helical (twisting of jet) as the jet moves downstream.

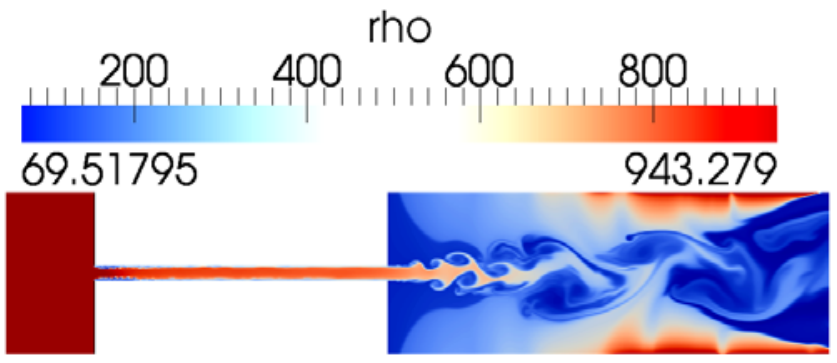

Figure 7: Density contour for $\mathrm{P}=5$ bar.

The phase change process results in vapour formation. Fig. 8 shows the mass fraction ( $\mathrm{x}$ ) and the volume fraction (alpha) for inlet pressure of 2 bar. Inside the core of the vortices, higher vapour formation is observed due to low pressure at the core of the vortices. Vapour formation takes place close to the nozzle exit because of the transfer of heat from the jet to the surrounding.

There is vapour formation downstream after the distance covered by the liquid jet. This is because of the pressure instability as the jet has not covered the whole numerical domain. This type of error is common in this kind of numerical problems (Gopalakrishnan [5]).

Increase in mass fraction and volume fraction takes place with the increase in the pressure ratio to 5 as shown in fig. 9. The higher momentum of the jet causes delay in significant vapour formation, which forms near the outlet of the domain compared to fig. 8 .

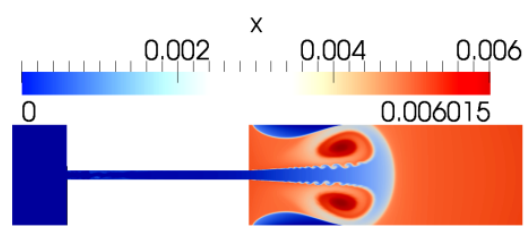

(a)

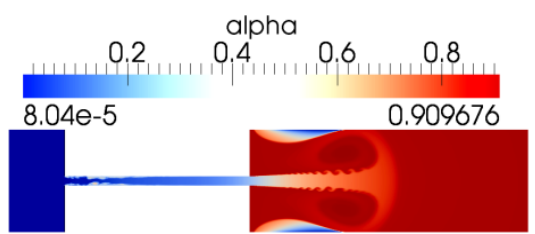

(b)

Figure 8: Contour for $\mathrm{P}=2$ bar (a) mass fraction (b) volume fraction.

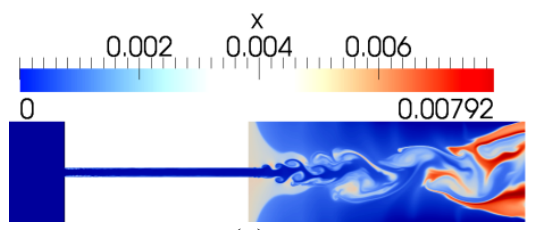

(a)

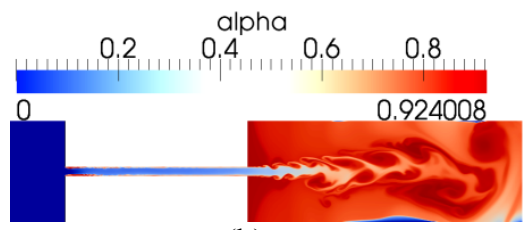

(b)

Figure 9: Contour for $\mathrm{P}=5$ bar (a) mass fraction (b) volume fraction. 
The jet travels more distance in fig. 9 because of higher speed compared to fig. 8 before it can diffuse the heat to the surrounding.

The centreline velocity and pressure profile for two inlet pressure ratios 2 and 5 are shown in fig. 10. There is an increase in the centreline velocity due to sudden decrease in pressure as the fluid comes out of the inlet. The presence of sharp corner creates flow separation, which leads to slight decrease in the centreline velocity. As the fluid travels through the nozzle, there is an exponential increase in the centreline velocity. A potential core is observed for inlet pressure of 2 bar, due to which almost constant velocity is maintained. Once this jet becomes fully developed, decrease in the centreline velocity is observed. The jet decreases exponentially after certain distance downstream and reaches almost close to zero velocity because the jet has not yet covered the complete domain. The centreline velocity is not zero at the end of the domain due to the induced velocity by the jet in the upstream. For inlet pressure 5 bar, there are huge amount of fluctuation in the centreline profile due to the instability of the jet, which is mainly helical in nature. There is a sudden drop of velocity near $0.15 \mathrm{~m}$ from the nozzle exit due to instability in the flow at that particular location.

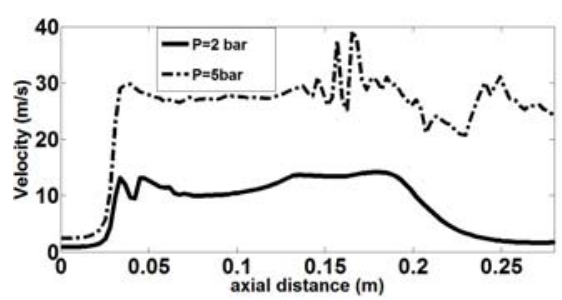

(a)

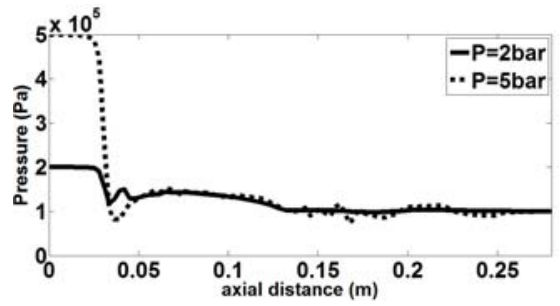

(b)

Figure 10: For inlet pressure 2 bar and 5 bar centreline profile for (a) velocity and (b) pressure.

The centreline pressure profile shows similar trend as observed in fig. 10(a). There is a sudden drop as the jet enters the nozzle. Inside the nozzle there is an exponential decrease in the pressure due to pressure drop and finally it reaches the outlet pressure value.

The drop in density is rapid for inlet pressure 5 bar compare to 2 bar before the nozzle exit as shown in fig. 11(a). For inlet pressure 5 bar, once the jet exits the nozzle, because of instability which causes high fluctuation in the density profile near axial distance $0.15 \mathrm{~m}$. This can be verified by the volume fraction profile, which shows a dip in the volume fraction at the same axial distance.

The velocity profile changes from Gaussian to top hat for both the inlet pressure for two axial distances $(0.008 \mathrm{~m}$ and $0.01 \mathrm{~m})$ are shown in fig. 12. The axial distance $0.008 \mathrm{~m}$ is close to the nozzle entrance, while the axial distance $0.01 \mathrm{~m}$ is inside the nozzle. The jet becomes fully turbulent inside the nozzle; this is due to the increase of velocity of the fluid inside the nozzle. The two double humps structure for inlet pressure 5 bar at an axial distance of $0.01 \mathrm{~m}$ are 
due to the shear interaction between the jet and the surrounding. The increase in velocity with the increase in axial distance is because of the acceleration of the jet inside the nozzle due to pressure drop.

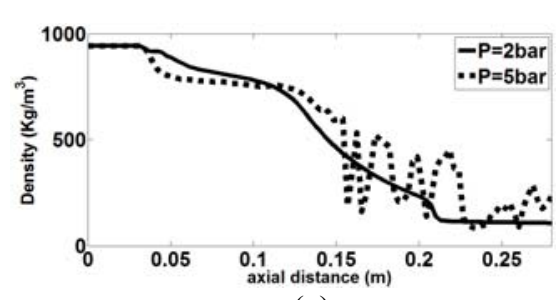

(a)

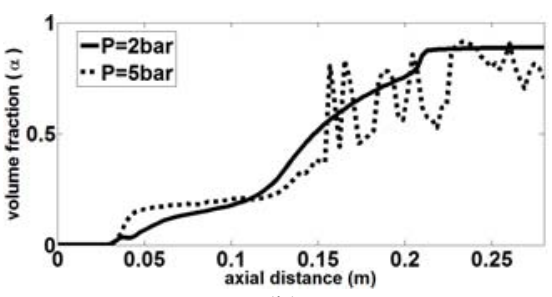

(b)

Figure 11: For inlet pressure 2 bar and 5 bar centreline profile for (a) velocity and (b) pressure.

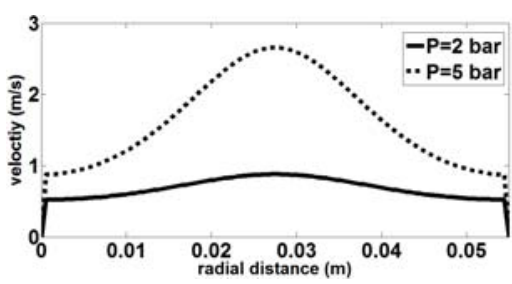

(a)

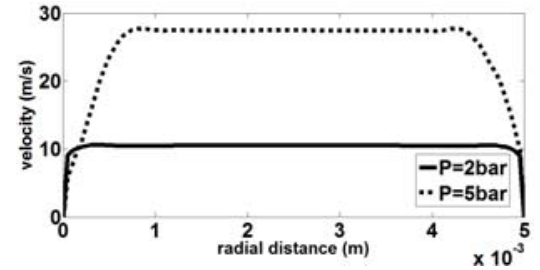

(b)

Figure 12: Velocity profile for inlet pressure of 2 bar and 5 bar from the inlet (a) $0.008 \mathrm{~m}$ and (b) $0.01 \mathrm{~m}$.

Dye visualization experiment was carried out for $\mathrm{P}=2$ bar at $393 \mathrm{~K}$ and the axisymmetric structure observed numerically was also observed in the experiment, downstream of the nozzle as shown in fig. 13. The temperature in the experiments varied within a range of $2 \%$. The instability in the jet decreases downstream of the nozzle and the jet expands due to the entrainment of the surrounding fluid. The semi cone angle obtained experimentally agrees well with the semi cone angle obtained numerically. The semi cone angle measured experimentally is $12.23^{\circ}$, while the semi cone angle obtained numerically is $11.3^{0}$. The values are well within the error limit.

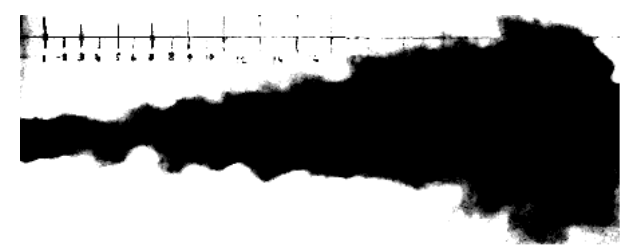

Figure 13: Axisymmetric structure observed for inlet pressure 2 bar at $\mathrm{T}=393 \mathrm{~K}$. 


\section{Conclusions}

The finite rate process of phase change was modelled successfully with HRM. The results obtained numerically for validation are in good agreement with that of experiment. With the increase in the inlet pressure, the acceleration of the jet increases inside the nozzle as well as outside the nozzle. The rapid decreasing of the fluid from high inlet pressure causes more formation of vapour and reduction in the density of the fluid. The velocity profile changes from Gaussian near the nozzle exit to top hat inside the nozzle, representing turbulent nature of the flow.

\section{References}

[1] Witlox, H.W., Flashing liquid jets and two-phase dispersion. Contract research report 404, 2002.

[2] Schmidt, D.P., Corradini, M.L., \& Rutland, C.J., A two-dimensional nonequilibrium model of flashing nozzle flow. Proc. of $3^{\text {rd }}$ ASME/JSME Joint fluid engineering conference, 1999, FEDSM.

[3] Schulz, W.D., Oxidation products of a surrogate JP-8 fuel. Symposium on structure of Jet Fuels III, 383-391, 1991.

[4] Spadaccini, L.J \& Huang, H., On line fuel deoxygenation for coke suppression. Transaction of the ASME 125, 686-692, 2003.

[5] Gopalakrishnan, S., Modelling of thermal non-equilibrium in superheated injector flows. Dissertation, UMAss Amherst, 2010.

[6] Allen, J.T., Laser based droplet size measurements in two-phase, flashing propane jets. Health and Safety Laboratory, 1996.

[7] Polanco, G., Holdo, A.E. \& Munday, G., General review of flashing jet studies Journal of Hazardous Materials, 2-18, 2010.

[8] Barret, Michel, Faucher, Eric \& Herand, Jean Marc., Schemes to compute unsteady flashing flows, AIAA Journal 40, 5, 2002.

[9] Ferziger, J.H. and Peric, M., Computational method for fluid dynamics, $3^{r d}$ ed Springer, 2002.

[10] Downar-Zapolski, P.Z., Bolle, L. \& Franco, J. The non-equilibrium relaxation model for one dimensional flashing liquid flow.IJMF22,473483, 1996. 